\title{
Nicht-steroidale Antiphlogistika erhöhen die Darmpermeabilität
}

\author{
I. O. Auer, W. Habscheid, S. Hiller, W. Gerhards und C. Eilles \\ Medizinische Universitätsklinik und Abteilung für Nuklearmedizin der Universität Würzburg
}

Zur Beantwortung der Frage, ob die unter Therapie mit nicht-steroidalen Antiphlogistika (NSA) erhöhte Urinausscheidung von oral aufgenommenem ${ }^{51} \mathrm{Cr}$ EDTA Ausdruck einer veränderten Darmpermeabilität ist, wurde die ${ }^{51} \mathrm{Cr}$-EDTA-Aktivität im Urin und Blut von Patienten unter NSA-Therapie sowie von Gesunden und kranken Kontrollen nach oraler Gabe von ${ }^{51} \mathrm{Cr}$-EDTA untersucht. Dabei ergab sich, daß die Urin- und Blut- ${ }^{51} \mathrm{Cr}$-EDTA-Aktivität von Patienten mit chronischer Polyarthritis $(\mathrm{n}=13)$ unter Therapie mit nicht-steroidalen Antiphlogistika signifikant höher waren als die einer Kontrollgruppe $(n=14)$, die sich aus Patienten mit chronischer Polyarthritis ohne NSATherapie $(n=9)$ und Patienten ohne rheumatische Erkrankungen $(n=5)$ zusammensetzte, und vergleichbar denen von Patienten mit Morbus Crohn $(\mathrm{n}=13)$. Bei Patienten mit chronischer Polyarthritis unter NSATherapie und Patienten mit Morbus Crohn bestand eine hochsignifikante Korrelation zwischen Urin- und Blutaktivität. Ein Einfluß der nicht-steroidalen Antiphlogistika auf die Nierenfunktion fand sich nicht. Diese Befunde lassen den Schluß zu, daß nichtsteroidale Antiphlogistika zu einer interenterozytären Permeabilitätssteigerung des Intestinaltraktes führen, deren Ausmaß mit der Permeabilitätsstörung beim Morbus Crohn vergleichbar ist.
Non-steroidal anti-inflammatory drugs increase intestinal permeability

${ }^{51} \mathrm{Cr}$-EDTA activity was measured in urine and blood of patients receiving non-steroidal anti-inflammatory (NSA) drug treatment and of healthy subjects and other patients (controls), after oral intake of ${ }^{51} \mathrm{Cr}$ EDTA, for the purpose of deciding whether NSA treatment increases urinary excretion of oral ${ }^{51} \mathrm{Cr}$-EDTA as an expression of increased intestinal permeability. ${ }^{51} \mathrm{Cr}$-EDTA activity in urine and blood of patients with rheumatoid arthritis (13) being treated with NSA was significantly higher (similar to results in 13 patients with Crohn's disease) than that of a control group (14) of patients with rheumatoid arthritis without such treatment (9) and patients without rheumatic disease (5). Both in patients with rheumatoid arthritis receiving NSA drugs and patients with Crohn's disease there was a highly significant correlation between urinary and blood activity. There was no effect of NSA drugs on renal function. The results indicate that NSA drugs increase interenterocytic permeability to an extent comparable to permeability abnormalities in Crohn's disease.
Der Gastrointestinaltrakt stellt mit $200-300 \mathrm{~m}^{2}$ die größte Grenzfläche zwischen menschlichem Organismus und Umwelt dar (34). Neben der Absorption ist die Aufrechterhaltung einer intakten Schrankenfunktion eine seiner Hauptaufgaben. Diese Schrankenfunktion oder Darmpermeabilität läßt sich als die Fähigkeit des Darmes definieren, den Ein- oder Durchtritt löslicher Substanzen in oder durch die Mucosa zu modifizieren (16). Die intestinale Aufnahme hängt von der Lipidlöslichkeit eines Moleküls, von aktiven oder erleichterten Absorptionsmechanismen für eine gelöste Substanz und von der Wasserlöslichkeit ab. In letzterem Falle liegt bei Fehlen aktiver oder erleichterter Absorptionsmechanismen eine nur sehr geringe Permeabilität vor. Niedermolekulare hydrophile Substanzen diffundieren möglicherweise durch definierte Kanäle der Zellmembran

Dtsch. med. Wschr. 112 (1987), 1032-1037

(c) 1987 Georg Thieme Verlag Stuttgart - New York ("aqueous pores «) in die Zelle (29), zum Beispiel Mannit und Polyethylenglykol 400. Größere hydrophile Moleküle können durch Zellmembranen nicht hindurchtreten und diffundieren in geringstem Umfang interzellulär (2). In den letzten Jahren sind verschiedene hydrophile Substanzen untersucht worden, die aufgrund ihres inerten Verhaltens im Gastrointestinaltrakt eine Aussage über eine Störung der Schrankenfunktion der Darmschleimhaut zu ermöglichen scheinen (16). Neben nieder- und hochmolekularem Polyethylenglykol wird wegen seiner einfachen Handhabung ${ }^{51} \mathrm{Cr}$-EDTA (Ethylendiamintetraacetat, Edetinsäure) bevorzugt eingesetzt.

Oral verabreichtes ${ }^{51} \mathrm{Cr}$-EDTA wird vom intakten Intestinum nur zu 1-2,5\% interenterozytär aufgenommen $(6,7,10,16,17)$. Nach Aufnahme wird es wegen seiner ausschließlich extrazellulären Lokalisation quantitativ schnell über die Nieren ausgeschieden $(6,7,10,17)$. Intakte Nierenfunktion vorausgesetzt, kann somit bei oraler Verabreichung der Testsubstanz aus der gemesse- 
nen Urinaktivität eine Aussage über deren gastrointestinale Aufnahme gemacht werden. Schrankenstörungen des Gastrointestinaltraktes wurden mit oral verabreichtem ${ }^{51} \mathrm{Cr}$-EDTA bei verschiedenen Erkrankungen festgestellt, so bei Coeliakie $(6,8-10)$, bei Neurodermitis und Nahrungsmittelallergien $(17,20)$, bei Morbus Crohn und Colitis ulcerosa $(7,25)$ sowie bei Patienten unter Zytostasetherapie (31). Auch der Genuß von Alkohol führt zu einer mit ${ }^{51} \mathrm{Cr}$-EDTA nachweisbaren Darmpermeabilitätsstörung.

Nicht-steroidale Antiphlogistika (NSA) verursachen zahlreiche unerwünschte Nebenwirkungen am Gastrointestinaltrakt (1). Bjarnason und Mitarbeiter (12) berichteten erstmals über eine erhöhte 24-Stunden-Urinausscheidung von oral aufgenommenem ${ }^{51} \mathrm{Cr}$-EDTA bei Patienten, die nicht-steroidale Antiphlogistika erhalten hatten, und interpretierten diesen Befund als Hinweis auf eine medikamentös induzierte Darmpermeabilitätsstörung. Diese Studien hatten jedoch die Möglichkeit einer Änderung der Nierenfunktion während oder durch die Untersuchung außer acht gelassen (32). Des weiteren fehlte der kritische Nachweis einer parallel zur Urinausscheidung ebenfalls erhöhten ${ }^{51} \mathrm{Cr}$-EDTA-Konzentration im Blut. Daher wurden Bjarnasons Ergebnisse und Interpretation in Zweifel gezogen $(24,32)$. Ziel unserer Untersuchungen war deshalb die Klärung der Frage, ob NSA Störungen der Darmpermeabilität bewirken. Dazu wurden folgende Aspekte untersucht:

1. Ist bei Patienten mit chronischer Polyarthritis bei Einnahme von NSA eine veränderte Urinausscheidung von oral verabreichtem ${ }^{51} \mathrm{Cr}$-EDTA im Vergleich zu Patienten mit chronischer Polyarthritis ohne NSA nachweisbar?

2. Geht einer gegebenenfalls geänderten ${ }^{51} \mathrm{Cr}$-EDTAAusscheidung im Urin eine Änderung der ${ }^{51} \mathrm{Cr}$-EDTAAktivität im Blut parallel?

3. Sind NSA-induzierte Änderungen der ${ }^{51} \mathrm{Cr}$-EDTAAusscheidung im Urin von Änderungen der glomerulären Filtrationsrate begleitet?

4. Läßt sich das Ausmaß einer eventuellen Permeabilitätsänderung durch NSA mit den bekannten Permeabilitätsstörungen beim Morbus Crohn vergleichen?

\section{Patienten und Methodik}

Zielgruppe. Sie bestand aus 13 Patienten, 9 Frauen und 4 Männern im mittleren Alter von 51,7 Jahren, mit gesicherter oder klassischer chronischer Polyarthritis (CP) nach den ARA-Kriterien bei Fehlen von Ausschlußkriterien. Bei acht Patienten war die Diagnose seit Jahren bekannt; von ihnen befanden sich zur Zeit der Untersuchung drei in Remission, fünf erhielten eine symptomatische Therapie mit NSA, drei davon eine Basistherapie mit parenteralem Gold. Bei fünf Patienten wurde kurz vor der Untersuchung die Diagnose der CP erstmals gestellt. Bei vier dieser Patienten wurde am Vorabend der ${ }^{51} \mathrm{Cr}$-EDTA-Verabreichung mit der NSA-Therapie begonnen; je zwei von ihnen erhielten Diclofenac (Voltaren ${ }^{3}$, 100 $\mathrm{mg} / \mathrm{d}$ ) und Indometacin (Amuno ${ }^{\circledR}, 100 \mathrm{mg} / \mathrm{d}$ ). Insgesamt wurden folgende nicht-steroidale Antirheumatika verabreicht: Diclofenac
(Voltaren) $100 \mathrm{mg} / \mathrm{d}(\mathrm{n}=3)$ und $250 \mathrm{mg} / \mathrm{d}(\mathrm{n}=2)$, Indometacin (Amuno) $100 \mathrm{mg} / \mathrm{d}(\mathrm{n}=3)$ und $150 \mathrm{mg} / \mathrm{d}(\mathrm{n}=2)$, Piroxicam $\left(\right.$ Felden $\left.{ }^{8}\right) 30 \mathrm{mg} / \mathrm{d}(\mathrm{n}=1)$ und $40 \mathrm{mg} / \mathrm{d}(\mathrm{n}=1)$ sowie $30 \mathrm{mg} / \mathrm{d}+$ Indometacin $100 \mathrm{mg} / \mathrm{d}(\mathrm{n}=1)$.

Die blutchemische Aktivität des Entzündungsprozesses der CPPatienten wurde mit Hilfe der Blutsenkungsreaktion sowie der Akute-Phase-Proteine $\alpha_{1}$-Antitrypsin und Haptoglobin bestimmt.

Kontrollgruppe. Sie setzte sich aus zwei Untergruppen (a und b) zusammen:

a) fünf Patienten im mittleren Alter von 54,8 Jahren ohne entzündliche rheumatische oder gastroenterologische Erkrankungen. Diagnosen: Mitralvitium $(n=1)$, transitorisch-ischämische Attacken $(n=1)$, Glutealabszeß $(n=2)$, Coxarthrose $(n=1)$,

b) neun Patienten im mittleren Alter von 46,9 Jahren mit entzündlich-rheumatischen Erkrankungen ohne NSA-Therapie. Acht dieser Patienten litten an einer gesicherten oder klassischen CP nach den ARA-Kriterien bei Fehlen von Ausschlußkriterien. Ein Patient hatte eine HLA-B27-positive Iliosakralarthritis. Bei fünf CP-Patienten war die Diagnose seit Jahren bekannt, davon befanden sich drei unter einer Basistherapie mit parenteralem Gold in Remission. Die anderen zwei Patienten erhielten bei Bedarf Paracetamol (ben-u-ron ${ }^{\circledR}$ ). Bei vier Patienten war die Diagnose erstmalig vor der Untersuchung gestellt worden. In zwei dieser Fälle wurde Paracetamol bei Bedarf verabreicht. Bei allen Patienten lag eine vormalige NSA-Therapie wenigstens drei Wochen zurück.

Patienten mit Morbus Crohn. Diese Gruppe bestand aus 13 Patienten im mittleren Alter von 28,6 Jahren mit histologisch gesicherter Erkrankung unterschiedlicher klinischer Aktivität, ermittelt mit Hilfe des Aktivitätsindex (CDAI) nach Best und Mitarbeitern (4). Bei je vier Patienten war ausschließlich das lleum bzw. Kolon befallen, bei fünf Patienten bestand eine Ileokolitis.

Verabreichung der NSA. Am Vorabend des Untersuchungstages erhielten die CP-Patienten der Zielgruppe die eine Hälfte der jeweiligen NSA-Tagesdosis und am Morgen des Testtages um $7^{30}$ Uhr, eine Stunde vor Einnahme von ${ }^{51} \mathrm{Cr}$-EDTA, die andere Hälfte.

${ }^{51} \mathrm{Cr}$-EDTA-Absorptionstest (10). Die nüchternen Patienten erhielten um $8^{30} \mathrm{Uhr}$ des Testtages $100( \pm 5) \mu \mathrm{Ci}{ }^{51} \mathrm{Cr}$-EDTA (Amersham Buchler, Braunschweig) in $10 \mathrm{ml}$ Wasser per os und tranken $300 \mathrm{ml}$ Leitungswasser nach. Danach folgte eine Nüchternperiode von zwei Stunden. Für den Testverlauf war der Genuß von Alkohol verboten. Blutentnahmen erfolgten zum Zeitpunkt 0 und eineinhalb, drei, sechs, zwölf und vierundzwanzig Stunden nach Aufnahme der Testsubstanz. Vor Testbeginn wurde die Blase entleert. Nach Testbeginn wurde der Urin in vier Fraktionen gesammelt: 0-6 Stunden, 6-12 Stunden, 12-24 Stunden, 24-48 Stunden. Im Gegensatz zu Bjarnason und Mitarbeitern $(10,12)$ war die Urinsammelperiode somit um 24 Stunden verlängert. Die geschätzte Strahlenbelastung während des Tests lag bei $<0,1 \mathrm{mSv}$. Die Proben (etwa $5 \mathrm{ml}$ Vollblut, $12 \mathrm{ml}$ Urin) wurden mit einem Gamma-Counter (Berthold, Frieshe BF, Gammaszint 5000) mit einer Meßzeit von 5 Minuten pro Probe, Meßtiefe $30 \mathrm{~mm}$, gemessen. Gleichzeitig wurden ein Nullwert und Probestandard pro Patient gemessen (ein Tausendstel der Gesamtaktivität, die der Patient zu sich nahm). Die ermittelten Aktivitäten wurden auf Prozent der applizierten Dosis mittels folgender Formel umgerechnet:

$\underset{\begin{array}{l}\text { Blutaktivität } \\ (\% \text { der Dosis })\end{array}}{\text { der }}=\frac{\text { Probe }_{\text {(netto) }} \times \text { Blutvolumen }_{\text {(gesamt) }}}{10 \times \text { Standardmittelwert }_{(\text {netro) }} \times \text { Probevolumen }}$

$\underset{(\% \text { der Dosis })}{\text { Urinaktivität }}=\frac{\text { Probe }_{\text {(netto) }} \times \text { Urinvolumen }}{10 \times \text { Standardmittelwert }_{\text {(netto) }} \times \text { Probevolumen }}$ 
Glomeruläre Filtrationsrate (GFR). Sie wurde mit der KreatininClearance $\left(\mathrm{C}_{\text {krea }}\right)$ (Urinsammelperiode 12 Stunden) ermittelt.

Statistik. Mangels Normalverteilung der Meßwerte der verschiedenen Patientengruppen erfolgte die statistische Auswertung mit Hilfe des U-Tests nach Mann und Whitney und des SpearmanRang-Korrelationstests. Der exakte Test nach Fisher kam mit der Yates-Korrektur zur Anwendung.

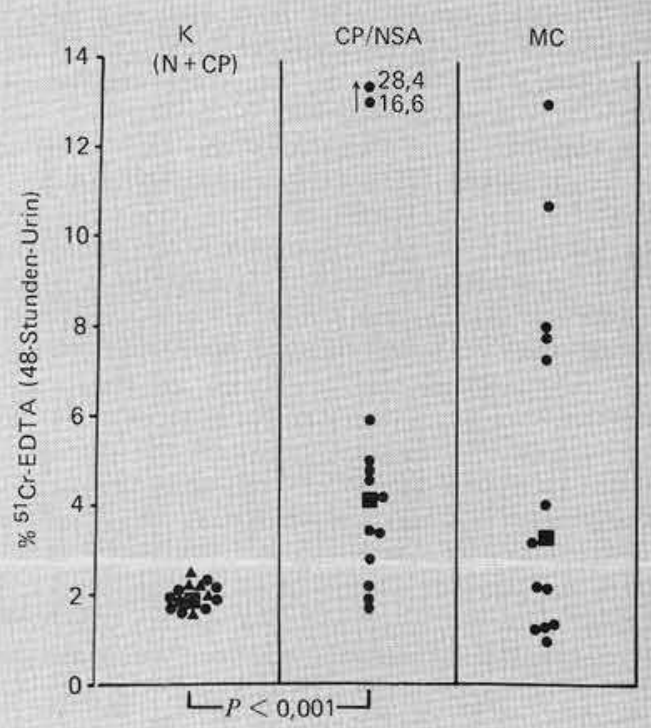

Abb. $1 .{ }^{51} \mathrm{Cr}$-EDTA-Ausscheidung im 48-Stunden-Sammelurin, ausgedrückt in Prozent der oral aufgenommenen Gesamt ${ }^{51} \mathrm{Cr}$-EDTAAktivität. Median, $\mathrm{K}=$ Kontrollgruppe, bestehend aus (A) Kontrollprobanden $\mathrm{N}$ und $(\mathrm{CP}$-Patienten ohne NSA, CP/NSA = Patienten mit chronischer Polyarthritis unter NSA, MC = Patienten mit Morbus Crohn.

\section{Ergebnisse}

${ }^{51} \mathrm{Cr}$-EDTA-Aktivität im Urin (Abbildung 1). Aus Strahlenschutzgründen und wegen der aus der Literatur bekannten, mehrfach reproduzierten Normalwerte der ${ }^{51} \mathrm{Cr}$-EDTA-Urinausscheidung nach oraler Gabe wurden nur fünf Kontrollprobanden ohne rheumatische oder gastrointestinale Erkrankungen untersucht. Die im Urin gemessene ${ }^{51} \mathrm{Cr}$-EDTA-Aktivität lag bei ihnen zwischen 1,5 und $2,5 \%(\bar{x}=2,00 \%)$ der oral verabreichten Dosis. Die ${ }^{51} \mathrm{Cr}$-EDTA-Aktivität im Urin der CP-Patienten ohne NSA ( $\mathrm{n}=9 ; \tilde{\mathrm{x}}=1,85 \%$ ) war derjenigen der Kontrollprobanden völlig vergleichbar. Darum war es berechtigt, Kontrollprobanden und CP-Patienten ohne NSA in einer Gruppe zusammenzufassen ( $\mathrm{n}=14 ; \overline{\mathrm{x}}=1,86 \%$ ).

Die ${ }^{51} \mathrm{Cr}$-EDTA-Aktivität im 48-Stunden-Sammelurin bei den 13 mit NSA behandelten CP-Patienten $(\overline{\mathrm{x}}=$ $4,10 \%)$ war signifikant höher $(P<0,001)$ als in der Kontrollgruppe. Hingegen unterschied sich diese Gruppe nicht signifikant von den Patienten mit Morbus Crohn $(\mathrm{n}=13 ; \overline{\mathrm{x}}=3,24 \%)$.

${ }^{51} \mathrm{Cr}$-EDTA-Aktivität im Blut (Abbildung 2). Ausgedrückt als Fläche unter der Blutspiegelkurve (AUC)

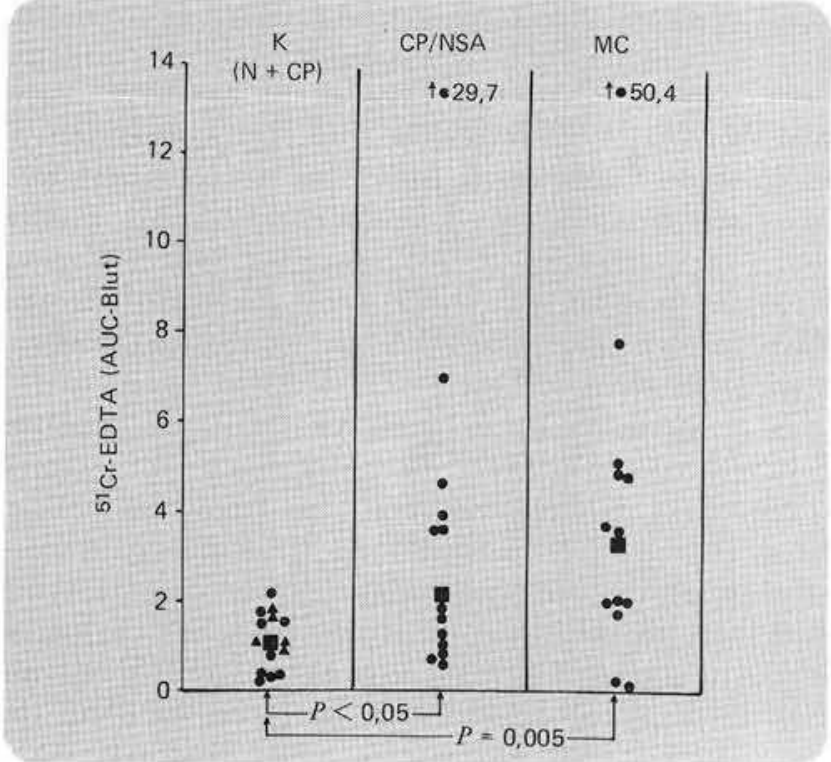

Abb. 2. ${ }^{51} \mathrm{Cr}$-EDTA-Aktivität im Blut, ausgedrückt als »area under the curve" (AUC), Meßwerte von 0 bis 24 Stunden. E Median, $K=$ Kontrollgruppe, bestehend aus $(\mathbf{A})$ Kontrollprobanden $\mathrm{N}$ und $(\mathbf{C P}$. Patienten ohne NSA, CP/NSA = Patienten mit chronischer Polyarthritis unter NSA, MC = Patienten mit Morbus Crohn.

waren die Werte der Kontrollprobanden $(\overline{\mathrm{x}}=1,24)$ und der CP-Patienten ohne NSA $(\overline{\mathrm{x}}=0,86)$ vergleichbar. Dagegen lagen sie bei den CP-Patienten unter NSA $(\overline{\mathrm{x}}=2,23)$ signifikant $(P<0,05)$ über denen der Kontrollgruppe $(\overline{\mathrm{x}}=3,24)$. Die Gruppe der Morbus-CrohnPatienten wies ihrerseits eine signifikant höhere ${ }^{51} \mathrm{Cr}$-EDTA-Aktivität im Blut auf als die Kontrollgruppe $(P=0,005)$.

Verhältnis der ${ }^{51} \mathrm{Cr}$-EDTA-Aktivität im 48-StundenUrin zur Aktivität im Blut (Abbildung 3). Die ${ }^{51} \mathrm{Cr}$ -

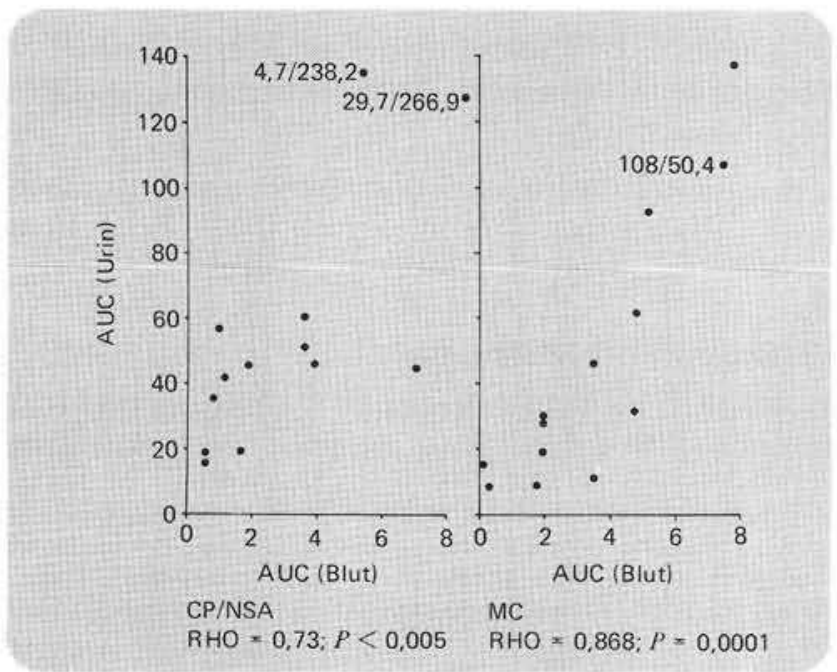

Abb. 3. Korrelation der ${ }^{51} \mathrm{Cr}$-EDTA-Urinausscheidung mit der ${ }^{51} \mathrm{Cr}$ EDTA-Aktivität im Blut, ausgedrückt als AUC. CP/NSA = Patienten mit chronischer Polyarthritis unter NSA, MC = Patienten mit Morbus Crohn. $\mathrm{RHO}=$ Rang-Korrelationskoeffizient nach Spearman. 


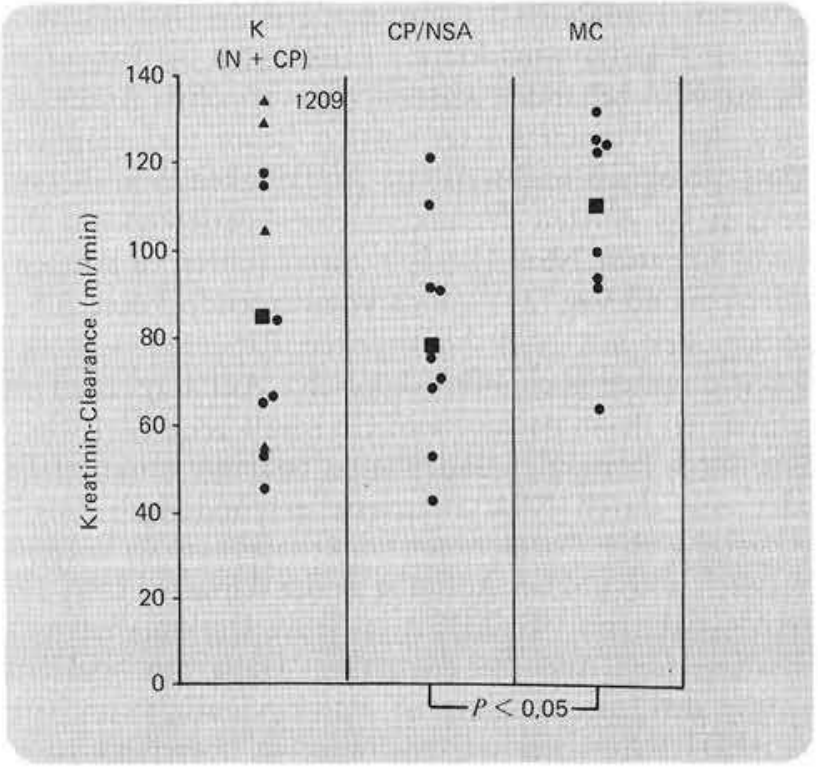

Abb. 4. Kreatinin-Clearance in den verschiedenen Gruppen. Median, $\mathrm{K}=$ Kontrollgruppe, bestehend aus ( $\mathbf{\Delta})$ Kontrollprobanden $\mathrm{N}$ und $(-\mathrm{CP}$-Patienten ohne NSA, CP/NSA = Patienten mit chronischer Polyarthritis unter NSA, MC = Patienten mit Morbus Crohn.

EDTA-Ausscheidung im Urin (Gesamtaktivität Urin sowie AUC) korrelierte hochsignifikant mit der ${ }^{51} \mathrm{Cr}$ EDTA-Aktivität im Blut (AUC Blut) bei den Patienten der Zielgruppe (Gesamtaktivität Urin/AUC Blut: $r=0,68 ; P<0,01$. AUC Urin/AUC Blut: $r=0,73$; $P<0,005)$ und den Morbus-Crohn-Patienten (Gesamtaktivität Urin/AUC Blut: $\mathrm{r}=0,79 ; P=0,001$. AUC Urin AUC Blut: $\mathrm{r}=0,868 ; P=0,0001)$.

Glomeruläre Filtrationsrate (Abbildung 4). Sie war bei den CP-Patienten unter NSA und der Kontrollgruppe vergleichbar. In beiden Gruppen war sie jedoch numerisch niedriger als bei den Morbus-Crohn-Patienten. Der Unterschied erreicht statistische Signifikanz beim Vergleich der CP-Patienten unter NSA mit den MorbusCrohn-Patienten $(P<0,05)$ und dürfte auf die unterschiedliche Altersstruktur der verglichenen Gruppen zurückzuführen sein.

Tab. 1. Abhängigkeit der ${ }^{51} \mathrm{Cr}$-EDTA-Aktivität im 48-Stunden-Sammelurin von der jeweils verabreichten NSA-Dosis

\begin{tabular}{|l|c|c|c|}
\hline \multirow{2}{*}{$\begin{array}{c}{ }^{51} \text { Cr-EDTA- } \\
\text { Ausscheidung* }\end{array}$} & nSA-Dosis* & Gesamt \\
\hline $\begin{array}{l}\text { niedrig } \\
\text { hoch }\end{array}$ & 7 & 1 & 8 \\
\hline Gesamt & 0 & 5 & 5 \\
\hline $\begin{array}{l}\text { * Dosishoch: }>100 \mathrm{mg} \text { Amuno; } \geqslant 200 \mathrm{mg} \text { Voltaren; } \geqslant 40 \mathrm{mg} \text { Felden } \\
\text { Ausscheidung hoch: Amuno }>5,2 \% ; \text { Voltaren }>3,2 \% ;\end{array}$ \\
\begin{tabular}{l} 
Felden $>2,5 \%$ \\
\hline
\end{tabular}
\end{tabular}

Abhängigkeit der ${ }^{51} \mathrm{Cr}$-EDTA-Aktivität im 48-Stunden-Urin von der NSA-Dosis. Tabelle 1 zeigt, daß mit der höheren Dosis eines jeweils verabreichten NSA eine signifikante Steigerung der Darmpermeabilität eintrat (exakter Test nach Fisher mit Yates-Korrektur; $P<0,005)$.

Tab. 2. Blutchemische Entzündungsaktivität in den verschiedenen Gruppen. $\mathrm{N}=$ Kontrollprobanden, $\mathrm{CP}$ ohne NSA = Patienten mit chronischer Polyarthritis (CP) ohne NSA. $\mathrm{K}=$ Kontrollgruppe, bestehend aus $\mathrm{N}+\mathrm{CP}$ ohne NSA, CP/NSA = Patienten mit chronischer Polyarthritis unter NSA, MC = Patienten mit Morbus Crohn

\begin{tabular}{|c|c|c|c|}
\hline & $\begin{array}{l}\text { Blutsenkungs- } \\
\text { reaktion } \\
(\mathrm{mm})\end{array}$ & $\begin{array}{l}\alpha_{1} \text {-Anti- } \\
\text { trypsin } \\
\text { (mg/dl) }\end{array}$ & $\begin{array}{l}\text { Hapto- } \\
\text { globin } \\
\text { (mg/dl) }\end{array}$ \\
\hline $\mathrm{N}$ & $13 \pm 4^{1,2}$ & $279 \pm 27$ & $127 \pm 21^{4,5}$ \\
\hline $\mathrm{CP}_{\text {ohne NSA }}$ & $30 \pm 9$ & $270 \pm 32$ & $200 \pm 39^{6}$ \\
\hline $\mathrm{K}$ & $24 \pm 6^{3}$ & $272 \pm 24$ & $182 \pm 31^{7.8}$ \\
\hline CP/NSA & $58 \pm 10^{1,3}$ & $327 \pm 24$ & $294 \pm 38^{4,7}$ \\
\hline MC & $40 \pm 8^{2}$ & $321 \pm 26$ & $305 \pm 24^{5,6,8}$ \\
\hline \multicolumn{2}{|c|}{ J-Test nach Mann und Whitney } & $\begin{array}{l}8 \\
3,3,5 \\
2,4,6,7\end{array}$ & $\begin{array}{l}005 \\
01 \\
05\end{array}$ \\
\hline
\end{tabular}

Blutchemische Krankheitsaktivität. Wie aus Tabelle 2 hervorgeht, waren Blutsenkungsreaktion und Haptoglobinkonzentration in der Gruppe der CP-Patienten unter NSA signifikant höher als in der Kontrollgruppe. Diese Unterschiede kamen jedoch im wesentlichen als Folge eines hochsignifikanten Unterschieds zwischen den CPPatienten unter NSA und den Normalkontrollen zustande, wohingegen zwischen CP-Patienten ohne und mit NSA keine signifikanten Unterschiede bestanden.

\section{Diskussion}

${ }^{51} \mathrm{Cr}$-EDTA ist eine wasserlösliche Substanz mit einer relativen Molekülmasse von 359. Es hat eine fischartige Konfiguration bei einer Länge von 1,26 nm und einer Breite von $0,9 \mathrm{~nm}$ (21). Trotz niedriger relativer Molekülmasse dringt es nicht durch die "aqueous pores " in die Zelle. Bei einer Bindungskonstanten von $10^{23}$ ( 9 ; im Vergleich: Bindungskonstante von Calcium-EDTA = $10^{11}$ ) ist das Molekül sehr stabil. Es geht keine Bindung mit den Plasmaproteinen ein und verbleibt, einmal aufgenommen, im Intravasalraum und wird zu $100 \%$ glomerulär filtriert (18). Die passive Aufnahme durch den Intestinaltrakt erfolgt im Gegensatz zu den Monosacchariden, die ebenfalls zur Darmpermeabilitätsmessung benutzt werden (15), durch interenterozytäre Diffusion (23). Eine Schädigung der Schrankenfunktion des Gastrointestinaltraktes bei chronisch entzündlichen Darmerkrankungen, bei Sprue, unter Alkoholeinnahme oder unter zytostatischer Therapie kann mit dem von Bjarnason und Mitarbeitern (10) beschriebenen und hier ange- 
wandten oralen ${ }^{51} \mathrm{Cr}$-EDTA-Test erkannt und möglicherweise quantifiziert werden.

Unsere Untersuchungen zur Darmpermeabilität bei Patienten mit chronischer Polyarthritis (CP) zeigen, daß bei CP-Patienten ohne NSA-Therapie die ${ }^{51} \mathrm{Cr}$-EDTAAusscheidung im Urin vergleichbar ist sowohl mit derjenigen von Patienten ohne rheumatische Erkrankungen als auch mit den aus der Literatur bekannten Werten größerer Gruppen gesunder Personen. Das macht deutlich, daß bei chronischer Polyarthritis eine ausgeprägtere, mit ${ }^{51} \mathrm{Cr}$-EDTA nachweisbare Darmpermeabilitätsstörung nicht vorliegt.

Als zweiten entscheidenden Befund ergaben unsere Untersuchungen in Bestätigung der Befunde von Bjarnason und Mitarbeitern (12) eine höhere Urinausscheidung von oral aufgenommenem ${ }^{51} \mathrm{Cr}$-EDTA bei Patienten mit CP unter NSA-Therapie als bei altersentsprechenden Kontrollprobanden, im wesentlichen CP-Patienten ohne NSA-Therapie. Dieser Befund ist nicht Folge einer NSAinduzierten Änderung der Nierenfunktion, wie von Simpson (32) eingewandt wurde, da die mit Hilfe der Kreatinin-Clearance bei unseren Patienten mit CP ohne und mit NSA gemessenen glomerulären Filtrationsraten vergleichbar waren.

Unsere Untersuchungen führen erstmals den Nachweis einer gesteigerten ${ }^{51} \mathrm{Cr}$-EDTA-Aktität im Blut nach Gabe von NSA. Bei den gesunden Kontrollpatienten und CPPatienten ohne NSA waren die Blutaktivitäten signifikant niedriger als bei den CP-Patienten unter NSA, bei denen eine hochsignifikante Korrelation zwischen der ${ }^{51} \mathrm{Cr}$-EDTA-Aktivität im Blut (AUC) und den gemessenen Urinaktivitäten bestand. Das läßt unter Berücksichtigung der unveränderten glomerulären Filtrationsrate den Schluß zu, daß die erhöhte ${ }^{51} \mathrm{Cr}$-EDTA-Ausscheidung unter NSA-Therapie tatsächlich durch eine medikamentös bedingte Steigerung der Darmpermeabilität verursacht ist.

Unsere Ergebnisse machen deutlich, daß die Steigerung der Darmpermeabilität abhängig ist von der Dosis des jeweils verabreichten NSA. Dabei werden bei den üblichen therapeutischen Dosen signifikante Erhöhungen der Darmpermeabilität beobachtet. Das Ausmaß der Permeabilitätssteigerung bei Patienten unter NSA ist vergleichbar dem bei Patienten mit Morbus Crohn. Im letzteren Fall ist das morphologische Substrat der erhöhten Permeabilität der entzündete Darm. Unklar ist hingegen der Pathomechanismus der NSA-induzierten Permeabilitätsstörung.

Aus älteren Versuchen an Ratten ist bekannt, daß es nach Gabe hoher NSA-Dosen zu perforierenden Ulzerationen im terminalen Ileum kommt (22). Robert (28) wies nach, daß bei gleichzeitiger Verabreichung von Prostaglandinen derartige Veränderungen vermieden werden können. Daß der zytoprotektive Effekt der Prostaglandine sich auch auf den unteren Intestinaltrakt erstreckt, läßt sich ebenfalls aus zahlreichen Fallberichten schließen (Übersichten: 1, 27). Bjarnason und Mitar-

beiter $(5,13)$ fanden bei szintigraphischen Untersuchungen mit ${ }^{111}$ Indium-markierten Leukozyten bei Patienten, die mit NSA behandelt wurden, eine vermehrte Anreicherung der Aktivität im terminalen Ileum und glaubten röntgenologisch nachweisbare Auffälligkeiten in diesem Bereich bei einigen der untersuchten Patienten auf die medikamentöse NSA-Therapie zurückführen zu können. Mielants und Veg (24) jedoch konnten endoskopisch bei keinem der mit NSA behandelten Patienten - weder makroskopisch noch mikroskopisch - Auffälligkeiten im terminalen Ileum nachweisen. Ob somit beim Menschen eine durch Prostaglandindepletion bedingte funktionelle oder eine durch NSA induzierte entzündungsbedingte Steigerung der Darmpermeabilität vorliegt, ist augenblicklich noch unklar. Auffällig ist der schnelle Effekt der NSA auf die mit ${ }^{51} \mathrm{Cr}$-EDTA meßbare Darmpermeabilitätssteigerung. Auch bei erstmaliger Gabe von NSA am Vortag der Untersuchung ist eine eindeutig vermehrte ${ }^{51} \mathrm{Cr}$-EDTA-Ausscheidung nachweisbar. In ersten Einzeluntersuchungen beobachteten wir bei intravenöser Gabe eines stabilen Prostacyclins (PG- $\mathrm{I}_{2}$ ) eine Normalisierung der NSA-induzierten, mit ${ }^{51} \mathrm{Cr}$-EDTA meßbaren Darmpermeabilitätssteigerung. Diese beiden letztgenannten Befunde sprechen eher für eine funktionelle, durch Prostaglandinsynthesehemmung hervorgerufene Störung der Darmpermeabilität.

Erstuntersuchungen von Bjarnason und Mitarbeitern (14) zufolge scheint Salazosulfapyridin die NSA-induzierte Darmpermeabilitätssteigerung zu normalisieren. Ob hierfür dessen modulierender Effekt auf die Produkte des Cyclooxygenaseweges $(3,19,26,30)$ oder der hemmende Effekt dieses Pharmakons auf den Lipoxygenaseweg (33) von Bedeutung ist, bleibt noch zu klären.

Inwieweit sich die hier mit Hilfe des ${ }^{51}$ Cr-EDTA belegte interenterozytäre Darmpermeabilitätssteigerung durch NSA auf andere großmolekulare Substanzen, Nahrungsantigene oder mikrobielle Antigene des Darminhaltes übertragen läßt, bedarf noch der Prüfung. Ebenfalls weiteren Untersuchungen bleibt es vorbehalten zu klären, inwieweit die hier belegte Darmpermeabilitätssteigerung durch NSA Einfluß auf die entzündlich-rheumatischen Erkrankungen selbst zu nehmen vermag.

\section{Literatur}

(1) Aucr, I. O.: Fortschritte und Perspektiven der Prophylaxe und Therapie gastrointestinaler Nebenwirkungen der medikamentösen Therapie mit nichtsteroidalen Antirheumatika, Akt. Rheum. (im Druck)

(2) Axon, A. T. R., B. Cracmer: The exsorption characteristics of various sugars. Gut $16(1975), 99$.

(3) Azad Khan, A. K., J. Piris, S. C. Truelove: An experiment to determine the active therapeutic moiety of sulphasalazine. Lancet 1977/11, 892.

(4) Bcst, W., J. M. Becktel, J. W. Singlcton, F. Kern jr.: Development of an

Crohn's disease activity index. Gastroenterology 70 (1976), 439.
(5) Bjarnason, J., J. Lang, M. J. Gumpcl A. J. Levi, A. B. Price: Non-steroidal antiinflammatory drugs (NSAID's) and intestinal pathology. Gastroenterology 90 (1986), 1947.

(6) Bjarnason, J., M. Marsh, A. Price, A. J. Levi, T. J. Petcrs: Intestinal permcability in patients with cocliac disease and dermatitis herpetiformis. Gut $26(1985)$ 1214.

(7) Bjarnason, J., C. O'Morain, A. J. Levi, T. J. Peters: Absorption of $\mathrm{Cr}^{51}$. labelled ethylencdiaminetetraacetate in inflammatory bowel disease. Gastroenterology 85 (1983), 318.

(8) Bjarnason, J., T. J. Peters: In vitro determination of small intestinal perme- 
ability. Demonstration of a persistent defect in patients with coeliac disease. Gut 25 (1984), 145.

(9) Bjarnason, J., T. J. Peters, N. Veall: Intestinal permeability defect in coeliac disease. Lancet 1983/, 1284.

(10) Bjarnason, J., T. J. Peters: A persistent defect in intestinal permeability in cocliac disease, demonstrated by $\mathrm{Cr}^{15}$. labelled EDTA absorption test. Lancet 1983/, 323.

(11) Bjarnason, J., K. Ward, T. J. Peters; The leaky gut of alcoholism. Possible route of entry for toxic compounds. Lan cet $1984 / 1,179$.

(12) Bjarnason, J., P. Williams, Alex So, G. D. Zanelli, A. J. Levi, J. M. Gumpel, T. J. Peters, B. Ansell: Intestinal permeability and inflammation in rheumatoid arthritis. Effects of non-steroidal anti-inflammatory drugs. Lancet 1984/II, 117.

(13) Bjarnason, J., P. Williams, G. Zanelli, P. Preuse, B. Ansell, T. J. Peters, J. M. Gumpel, A. J. Levi: Non-steroidal antiinflammatory drug (NSAID) induced ileocoecal inflammation in man. Gastroenterology 88 (1985), 1327.

(14) Bjarnason, J., G. Zanelli, P. Prouse, P. Williams, J. M. Gumpel, A. J. Levi: Non-steroidal anti-inflammatory drug (NSAID) induced intestinal inflammatio in man. Treatment. Gastroenterology 90 (1986), 1348
(15) Cobden, I., J. Rothwell, T. R. Axon: Intestinal permeability and screening tests for coeliac disease. Gut 21 (1980), 512.

(16) Editorial: Intestinal permeability. Lancet 1985/1, 256.

(17) Forget, P., F. Sadoyez-Goffaux, A. Zappitelli: Permeability of the small intestine to $\mathrm{Cr}^{51}$ EDTA in children with acute gastroenteritis or eczema. J. pediat. Gastroent. 4 (1985), 393.

(18) Garnett, E. S., V. Parson, N. Veall: Measurement of glomerular filtration rate in man using $\mathrm{Cr}$-51-EDTA complex. Lancet 1967/1, 818.

(19) Hillier, K., P. Mason, C. L. Smith: Ulcerative colitis. Prostaglandin metabolism and the effect of sulphasalazine, Saminosalicylic acid and indomethacine in human colonic mucosa. Brit. J. Pharmacol. 73 (1981), 217.

(20) Jackson, P. G., M. H. Lessof, R. Baker, J. Ferret, M. McDonald: Intestinal permeability in patients with eczema and food allergy, Lancet 1981/I, 1285.

(21) Jenkins, R. T., C. J. L. Lock, J. Roony: $\mathrm{Cr}^{51}$ EDTA in studies of intestinal permeability. Lancet 1984/II, 1342.

(22) Kent, T. H., R. Cardelli, F. W. Stamler: Small intestinal ulcus and intestinal flora in rats given indomethacin. Amer. J. Path. 54 (1969), 237.
(23) Lokken, P.: Studies on Cr-51-EDTA and its Evaluation as a Reference Substance in Gastrointestinal Research. Norwegian Monographs in Medical Science (Universitetsforlagett: Oslo 1970). (24) Mielants, H., E. M. Veg: NSA and the leaky gut. Lancet 1985/I, 218.

(25) Peled, Y., C. Watz, T, Gilat: Measurement of intestinal permeability using $\mathrm{Cr}$-51-EDTA. Gastroenterology 80 (1985), 770.

(26) Rampton, D. S., C. J. Hawkey: Prostaglandines and ulcerative colitis. Gut 25 (1984), 1299.

(27 Ritschard, Th., L. Filippini: Nebenwirkungen nichtsteroidaler Antiphlogistika auf den unteren Intestinaltrakt. Disch. med. Wschr. 111 (1986), 1561.

(28) Robert, A.: An intestinal disease produced experimentally by a prosta glandin deficiency, Gastroenterology 69 (1975), 1045.

(29) Salomon, A. K., B. Chasan, J. A. Dix, M. F. Lukakovic, M. R. Toon, R. Veikman: The aqueous pore in the red cell membrane as a channel for electrolytes and water. Ann. N. Y. Acad. Sci. (1984), 414.

(30) Schlenka, Th., W. Guck, B. M Peshar: Effekt von Sulphosalazin auf das Prostaglandinsystem menschlicher Kolonmukosa. Z. Gastroent. 19 (1981), 526.

(31) Selby, P., T. J. McElwain, M. Crofts, N. Lopes, I. Mundy: Cr-EDTA test for intestinal permeability. Lancet 1984/, 38.

(32) Simpson, L. O.: NSA and the leaky gut. Lancet 1985/, 218.

(33) Stenson, W. F., B. Lobos: Sulfasalazine inhibits the synthesis of chemotactic lipoids by neutrophils. J. clin. Invest. 69 (1982), 494.

(34) Wilson, T. H.: Intestinal Absorption (Saunders: Philadelphia 1968).

Prof. Dr. I. O. Auer, Dr. W. Habscheid, cand. med. S. Hiller Medizinische Universitätsklinik

Dr. W. Gerhards, Dr. C. Eilles

Abteilung für Nuklearmedizin

Klinik und Poliklinik für Strahlentherapie

der Universität

8700 Würzburg, Josef-Schneider-Str. 2 